\title{
Inferring the nature of turbulence in star-forming regions with polarimetric observations
}

\author{
D. Wiebe ${ }^{1}$ and W. D. Watson ${ }^{2}$ \\ ${ }^{1}$ Institute of Astronomy of the RAS, Moscow, Russia \\ email: dwiebe@inasan.ru \\ ${ }^{2}$ University of Illinois at Urbana-Champaign, USA \\ email:w-watson@uiuc.edu
}

\begin{abstract}
We consider the possibility to study the nature of MHD turbulence in star-forming regions with three different kinds of polarimetric data, namely, the linear polarization of starlight due to extinction by aligned dust grains, the polarized dust thermal radiation at far infrared or submillimeter wavelengths, and the linear polarization of molecular lines due to the GoldreichKylafis effect in the anisotropic MHD medium.
\end{abstract}

Keywords. ISM: magnetic fields, turbulence, polarization

The starlight polarimetry relates properties of the tenuous interclump medium to properties of relatively dense clumps within molecular clouds. The observed lack of contribution of dark clumps to the polarization of background stars is readily reproduced if the magnetic field structure in these clumps is less regular than in the interclump medium and the number of turbulence correlation lengths along the LOS within the clump is about 10 or more. The essential component of the model is that starlight that enters the clump is already polarized (Wiebe \& Watson 2001).

Quite irregular magnetic fields in dense clumps, implied by the starlight polarimetry data, still may provide enough alignment in the dust grain ensemble, so that thermal dust emission is polarized at a level of a few per cent. We use a turbulent polarization reduction factor $F$ to describe the influence of the irregular component of magnetic field on the observed fractional polarization. The presence of irregular magnetic field, which is 1.5 stronger than average field, decreases percentage polarization by an order of magnitude. This is compatible with most observations (Wiebe \& Watson 2001).

The intrinsic anisotropy of MHD turbulence may also result in an observed polarization of thermal molecular lines. We calculate the linear polarization for the microwave lines of the $\mathrm{CO}$ molecule in the anisotropic MHD medium. The maximum degree of polarization occurs at a column density of $10^{15} \mathrm{~cm}^{-2}$ which corresponds roughly to $\tau \sim 1$. The fractional polarization reaches $8 \%$ at $C / A=0.1$ for the strong magnetic field $\left(V_{\mathrm{A}} / c_{s}=\right.$ $10)$. When the average magnetic field is weak, the fractional polarization also is smaller, indicating less anisotropy in the medium (Wiebe \& Watson, ApJ, accepted).

\section{Acknowledgements}

D. W. is supported by the RFFI grant 04-02-16637.

\section{References}

Wiebe, D. \& Watson, W.D. 2001, ApJ 549, L115

Wiebe, D. \& Watson, W.D. 2004, ApJ 615, 300 